Wiı.t.M J. M. LliveLT (Nijmegen)

\title{
Zur sprachlichen Abbildung des Raumes: Deiktische und intrinsische Perspektive ${ }^{1}$
}

Elwas über räumliche Verhältnisse zu sagen, kann ausgesprochen schwierig sein. Es ist schon nicht einfach, einen Weg zu beschreibeu. Noch weniger einfach aber ist es, eine solche Beschreibung zu verstehen. Jeder von uns kann sich selbst dabei beobachten, daf ir gelegentlich rechts und links verwechselt. Niemandem gelingt es wirklicin, auf Grund einer sprachlichen Beschreibung ein getreues inneres Bild von einem Haus oder einer Wohnung zu entwickeln, wie lebendig die Beschreibung auch sein mag.

Diese Schwierigkeiten haben ihre guten Gründe. Räumliche Repräsentationen unterscheiden sich grundsätzlich von sprachlichen Reprïsentationen, und das Reden über räumliche Verhältnisse erfordert clie Projektion der räumlichen auf die sprachlichen Repräsentationen. Die mentale Repräsentation des Raumes ist dreidimensional, lokal euklidisch, topologisch (vgl. Wunderlich in diesem Band); sie wird manchmal als analog bezeichnet. Sprachliche Repräsentationen dagegen sind geordnete eindimensionale Ketten diskreter Elemente; es handelt sich dabei um deklarative Strukturen, mit denen Prädikationen, Propositionen und andere kategorische Strukturen ausgedrückt werden. Der Weg, auf dem Sprachbenutzer von analogen zu deklarativen Reprösentationen gelangen können, ist in keiner Weise strikt determiniert, Vielmehr besteht hier ein beträchtliches $\mathrm{Maß}$ an Freiheitsgraden. Sprachbenutzer kötanen eine unterschiedliche Perspektive bezüglich einer räumlichen Gegebenheit einnehmen, und jede Perspektive führt zil teiner ihr eigenen Art der Abbildung räumlicher Verhältnisse in deklarativer Form.

1 Dic Grundlage dieses Aufsatzes waren perzeptuelle und sprachliche Intuitionen. Thnen sollte man mit MiBtrauen begegnen. Melissa Bowerman hat mich auf einige zweifelhafte Fälle einer vorherigen Version hingewiesen und mich durch die Konstruktion einiger äußerst überzeugender Gegenbeispiele gezwungen, dje Theorie zu verïndern. Weitere hilfreiche Kommentare verdanke ich Emmon Bach, Herb Clark und den Mitgliedern der Gruppe Sprachproduktion unseres Institutes. Inge Tarim hat alle Abbildungen gezeichnet. Ein leicht veränderte und ausführlichere Fassung dieses Aufsatzes wurde veröffentlicht in The limits of perception, herausgegeben von A. van Doorn, W. A. van de Grind, und J. J. Koenderink, Utrecht: VNU, 1984. Der Texl wurde von Thomas Pechmann ins Detitsche übersetzt. 
Die vorliegende Arbeit befaßt sich mit zwei Möglichkeiten, eine Perspektive einzunehmen: mit der deiktischen und der intrinsischen Perspektive. Dies sind keineswegs die einzigen, die einem Sprecher oder Hörer zur Verfügung stehen, aber sie sind möglicherweise universal in den Sprachen und Kulturen der Welt anzutreffen. Die Untersuchung wird darauf beschränkt sein, welche Rolle diese Perspektiven bei dem Gebrauch von drei Paaren sprachlicher Ausdrücke spielen: links/ rechts, vor/hinter und über/unter. Anhand dieser Ausdrücke ist es möglich, einige der grundlegenden formalen, perzeptuellen und funktionalen Eigenschaften dieser Perspektivsysteme zu verdeutlichen, die bei der deklarativen Repräsentation der drei wichtigsten Dimensionen räumlicher Erfahrung von Bedeutung sind.

\section{1. 'Links' und ,Rechts', deiktischer und intrinsischer Gebrauch.}

Es ist einfach, mit links' und, rechts' Verwirrung zu stiften. Betrachten wir zunächst die Abb. 1a. Wenn man den vorliegenden Text zum Lesen vor sich hat, kann es keinen Zweifel daran geben, daß der mit , $\mathrm{A}^{2}$ bezeichnete Ball sich links von dem mit , $B^{4}$ bezeichneten $B a l l$ befindet und đaß $B$ rechts von $A$ ist.

(a)

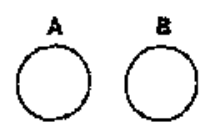

v (b)

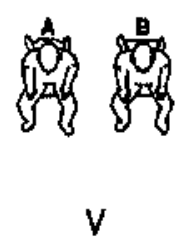

(c)

$\wedge$

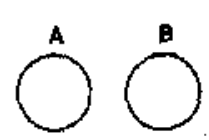

(d)

$\wedge$

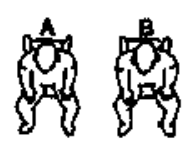

Abb. 1: Deiktischer und intrinsischer Gebrauch von ,links` und ,rechts

Mañ drückt sich ein wenig genauer aus, wenn man sagt, daß die zuletzt. gemachte Aussage für den Fall gilt, daß sich der Betrachter in der Position V der Abbildung befindet und damit A und B frontal vor sich hat. Im folgenden wird $\mathrm{V}$ als Symbol benutzt um anzuzeigen, in welche Richtung die Augen des Betrachters blicken. Wir werden außerdem von der folgenden terminologischen Konvention Gebrauch machen: in den zu analysierenden Fällen wird es immer ein ,Verweisobjekt' und ein ,Bezugsobjekt' geben. In der Äußerung , $A$ ist links von $B^{\prime}$ beispielsweise ist $A$ das Verweisobjekt und $B$ das Bezugsobjekt. In , B ist rechts von $A^{c}$ ist $B$ das Verweisobjekt, und $A$ ist das Bezugsobjekt. Die jeweilige Rolle der beteiligten Objekte wird man in der Regel aus dem Kontext ableiten können, aber dann und wann wird eine explizite Terminologie notwendig sein. 
Wenn wir nun zur Abbildung 16 übergehen, können wir die ersten Probleme mit ,links' und, rechts' beobachten. Wie bezüglich Abbildung ia kann der Betrachter der Abbildung 1b korrekterweise sagen, daß $B$ rechts von $A$ ist, aber es ist auch nicht falsch $z u$ sagen, da $B$ B sich links von $A$ befindet, da $B$ zur linken Hand von $A$ sitzt. Demnach befindet sich B sowohl links als auch rechts von A. Ist der Gebrauch dieser Ausdrücke abhängig von der Position des Betrachters? Um das zu testen, betrachten wir nun Abbildung 1c. Der Betrachter der Abbildung 1a ist nun um die Bälle $A$ und $B$ herumgegangen und betrachtet sie sozusagen von hinten. Aus dieser Perspektive befindet sich $B$ eindeutig links von $A$, und $A$ ist rechts von $B$. Mit anderen Worten, die Beziehungen haben sich hier im Vergleich zur Abbildung 1 a genau umgekehrt. Die Veränderung der Perspektive des Betrachters hat einen EinfluB auf den Gebrauch von, links' und, rechts'. Wenn der Betrachter der Abbildung $1 b$ so um $A$ und $B$ herumgeht, daB er sich schließlich in der in Abbildung 1d wiedergegebenen Position befindet, löst sich die Mehrdeutigkeit des Gebrauchs von ,links" und ,rechts" auf. $B$ ist links von $A$, und $A$ ist rechts von $B$. Es gibt keine andere Möglichkeit.

Die Ursachen für die beschriebenen Unterschiede und Mehrdeutigkeiten sind hinreichend bekannt. Sprachbenutzer haben (mindestens) zwei Systeme, um auf räumliche Beziehungen zu verweisen, das deiktische System und das intrinsische System. Wenn ein Sprecher das deiktische System benutzt, interpretiert er räumliche Ausdrücke in bezug auf seine eigene Perspektive. In den Abbildungen 1a und 1c wird ausschließlich dieses System benutzt. Wenn sich die Perspektive des Sprechers ändert, ändert sich entsprechend auch die Wahl von ,links' und, rechts'. Man beachte, daß ein Hörer, der mit der räumlichen Beschreibung des Sprechers konfrontiert wird, diese nicht korrekt interpretieren kann, ohne zu wissen, welche Position der Sprecher in der jeweiligen Situation einnimmt. Das deiktische System dürfte vor allem für kommunikative Situationen geeignet sein, in denen die Gesprächspartner wissen, welche Perspektive jeder von ihnen einriimmt. Im folgenden werden wir allerdings davon ausgehen, daß der Betrachter bzw. Sprecher sich nicht vom Hörer unterscheidet, was seine relative Position und Orientierung betrifft. Es mag zwar vorkommen, daß der Gebrauch räumlicher Ausdrücke in bestimmten Situationen mit den räumlichen Beziehungen zwischen Sprecher und Hörer variiert. Darauf wird hier jedoch nicht eingegangen.

Wenn wir nun zu den Abbildungen $1 \mathrm{~b}$ und $1 \mathrm{~d}$ übergehen, dann ist zunächst zu bemerken, daß der Betrachter auch in diesen Fällen das deiktische System verwenden könnte. Er würde dann dieselben Ausdrücke benutzen wie bei der Betrachtung der Abbildungen 1a und 1c. Aber es gibt eine weitere Möglicheit beztiglich der Abbildungen 1b 
und 1d, nämlich die Verwendung des intrinsischen Systems. In diesem referentiellen System werden räumliche Ausdrücke relativ zur intrinsischen Orientisrung oder Perspektive der Bezugsobjekte selbst interpretiert. In den Abbildungen $1 \mathrm{~b}$ und $1 \mathrm{~d}$ befindet sich die Person $\mathrm{A}$ in einer Position, in der B von ihr selbst aus gesehen sich links befindet. Diese Orientierung ist von der Perspektive des Betrachters unabhängig; dites gilt sowohl für $1 \mathrm{~b}$ als auch für $1 \mathrm{~d}$. Um das intrinsische System zu verwenden, muB der Sprachbenutzer in der Lage sein, die Situation zu interpretieren. Er muß wissen, was für Objekte in der Situation vorkommen. Wenn der Betrachter der Abbildung 1b wenige Augenblicke vor dem Experiment seine Brille verlieren würde, wäre er hicht in der Lage zu sehen, daB A und B zwei sitzende Personen sind. Sollte er A als einen Sack Kartoffeln wahrnehmen, könnte er nicht äußern, daß B links von A ist. Seine einzige Möglichkeit wäre, das deiktische System zu benutzen und zu sagen, daß B rechts von $A$ ist. Das deiktische System kann immer benutzt werden, es ist das Standardsystem, aber es erfordert, daß der Hörer weiß, wo sich der Sprecher befindet. Das intrinsische System verlangt nicht nur eine Interpretation der Situation, es kann darüber hinaus nur dann verwendet werden, wenn es Bezugsobjekte mit einer intrinsischen Orientierung gibt, wie z. B. Personen, Autos, Kirchen, Stühle, Telefone usw. Es kann nicht auf Bälle, runde oder viereckige Tische, Bäume, Sandhaufen angewendet werden (zumindest nicht bei Ausdrücken wie ,links' und ,rechts", ,vor" oder ,hinter'). Dies ist der Grund, warum die Objekte A und B in den Abbildungen 1a und 1c als Kreise gezeichnet wurden; sie erlauben dadurch keine intrinsische Interpretation.

Die in bezug auf Abbildung $1 \mathrm{~b}$ beobachtete Mehrdeutigkeit ist damit erklärbar. Das deiktische System und das intrinsische System stehen in vollem Gegensatz zueinander. Der Konflikt löst sich allerdings auf, wenn der Betrachter $\mathrm{zu}$ der in Abbildung 1d angegebenen Position übergeht. Aus dieser Position ist B sowohl aus der Perspektive des Betrachters (deiktisches System) als auch bezüglich der Orientierung von $A$ (intrinsisches System) stets links von $A$.

Diese Duplizität von Systemen räumlicher Referenz gilt sehr allgemein für die Sprachen auf der Welt, obwohl es im Detail viele Unterschiede gibt (vgl. u. a. Weissenborn \& Klein, 1982). Das führt zu einer Reihe wichtiger Fragen. Die schwierigste ist, warum die Evolution der Sprache zu dieser Duplizität geführt hat. Dienen die beiden Systeme unterschiedlichen Funktionen? Und haben sich beide Systeme a.us einem gemeinsamen Ursprung entwickelt, der phylogenetisch oder ontogenetisch vor ihrer getrennten Entwicklung anzusetzen ist? Ein zweiter Punkt betrifft die Frage, wie Sprachbenutzer mit möglichen Verwechslungen beider Systeme umgehen. Wie kann ein Hörer wissen, welches System der Sprecher verwendet? Gibt es irgendwelche perzep- 
tuellen oder sprachlichen Merkmale, die den Hörer darauf hinweisen, welches System gerade benutzt wird? In der vorliegenden Arbeit werden wir uns hauptsächlich auf einen dritten Fragenkomplex konzentrieten: gibt es irgendwelche markierte formale Unterschiede zwischen diesen Systemen der Perspektivïbernahme, und welche perzeptuellen und funktionalen Bedingungen müssen bei ihrer adäquaten Verwendung erfüllt sein?

2. Umkehrbarkeit und Transitivität bei deiktischem gegenüber intrinsischem Gebrauch von, links' und, rechts"

Stehen, links' und ,rechts' in einer Umkehr-Beziehung zueinander, d. fl. ist $B$ immer rechts von $A$, wenn $A$ links von $B$ ist? Es gilt zu beachten, $\mathrm{da} B$ der Eigenschaft der Umkehrbarkeit eine wesentliche Bedeutung bei der Verwendung räumlicher Begriffe zukommt. Sie erlaubt uns, die Beziehung, links von' aus der Beziehung, rechts von' und umgekehrt zu erschließen.

Umkehrbarkeit gilt nur für das deiktische System. Die Perspektive des Betrachters in der Abbildung 1a, die in der Abbildung 2a noch einmal wiedergegeben wurde, garantiert, $d a B B$ rechts von $A$ ist und daß $A$ links von $B$ ist. Von einem feststehenden Punkt der Betrachtung aus gesehen ist nicht möglich, daß die eine Beziehung zutrifft, ohne $\mathrm{da} B$ die andere Beziehung ebenfalls zutrifft.

(a)<smiles>c1ccccc1</smiles>

$\langle c\rangle$<smiles>[C]1CCCCC1</smiles><smiles>[C]1CCCCC1</smiles><smiles>[C]1CCCCC1</smiles>

V

2 (c) $A$ ist links von $B$ $B$ ist links von $C$

A ist links von $\mathrm{C}$ (b)

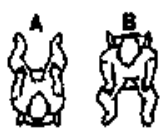

(d)

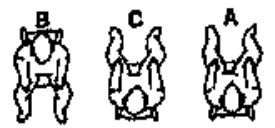

2 (d)

A list links von $B$

$B$ ist links von $C$

A ist rechts von $\mathrm{C}$

Abb, 2: Umkehrbarkeit und Transitivität bei dejktischem und intrinsischem Gebrauch von, tinks" und, rechts'. 
Aber vergleichen wir dies mit der Situation der Abbildung $2 b$. In dieser Situation ist eine intrinsische Interpretation möglich (und um deutlich zu machen, daß dies die Interpretation ist, um die es uns in diesem Fall geht, haben wir den Betrachter in der Abbildung weggelassen, weil dessen Position für das intrinsische System irrelevant ist). B ist in dieser Abbildung rechts von A, aber A ist nicht links von B. Vielmehr ist $A$ rechts von B. Damit sind A und B jeweils rechts voneinander; die Beziehungen sind also nicht umkehrbar. Eine detaillierte Analyse umkehrbarer Beziehungen, dort ,Antonymität ' genannt, enthält Ehrich (1985).

Wie verhält es sich nun mit der Transitivität? Die beiden Système unterscheiden sich auch in dieser Beziehung. Das deiktische System ist lokal transitiv, wie man aus der Abbildung $2 c$ ersehen kann. Aus der Perspektive des Betrachters ist A links von B, und B ist links von C. Es trifft dann notwendigerweise auch $\mathrm{zu}$, daß $\mathrm{A}$. links von $\mathrm{C}$ ist. Aber das intrinsische System ist nicht transitiv. Wenn die Abbildung 2d intrinsisch interpretiert wird, $d$.h. unabhängig von der Perspektive eines Betrachters, dann ist A links von B, B ist links von C, aber A ist nicht links von $\mathrm{C}$. A ist vielmehr rechts von $\mathrm{C}$.

Es ist bisher in der Literatur überhaupt nicht beachtet worden, $\mathrm{da} B$ das intrinsische System lokal intransitiv ist, und das hat $\mathrm{zu}$ beträchtlicher Konfusion geführt. Wenn Johnson-Laird (1980, S. 87) die Transitivität von ,rechts' diskutiert, geht er dabei stillschweigend vom deikisschen zum intrinsischen System über und bemerkt dann, daß die

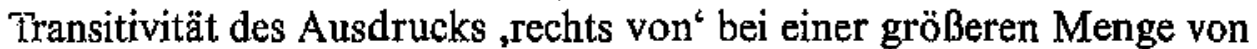
Objekten wie beispielsweise bei Personen, die um einen großen runden Tisch sitzen, nicht mehr zutrifft, und genau dieselbe Überlegung wird in Johnson-Laird (1983, S.240 f.) wiederholt. Tatsache ist jedoch, daß das intrinsische System noch nicht einmal lokal transitiv ist, wie man der Abbildung 2d entnehmen kann, während das deiktische System vollständig transitiv ist, wenn man sich einen Betrachter vorstellt, der den runden Tisch aus einer gewissen Entfernung betrachtet.

Eine Folge der lokalen Intransitivität des intrinsischen Systems ist, da $B$ der Umgang mit, links' und ,rechts' in diesem System insgesamt schwierig ist. Wenn man eine Person bitten würde, die korrekte räumliche Anordnung auf Grund der Beschreibung „Hans ist links von Maria, Maria ist links von Peter, und Hans ist rechts von Peter" zu zeichnen, $\mathrm{d}, \mathrm{h}$. die räumlichen Beziehungen wiederzugeben, die die Abbildung $2 \mathrm{~d}$ darstellt, dann wird diese Person eine relativ lange Zeit benötigen, um die korrekte Lösung zu finden (wahrscheinlich gelangt sie zu der quasi-Lösung, die drei Personen um einen runden Tisch zu gruppieren). 
3. Wahrnehmung und Wissen beim Gebrauch der deiktischen und intrinsischen Perspektive

Das deiktische System erfordert die Perspektive eines Betrachters, und man kann sich fragen, welchen einschränkenden Bedingungen diese Perspektive unterliegt. In der Abbildung $3 \mathrm{a}$ steht der Betrachter aufrecht und beobachtet zwei Ballons am Horizont. In diesem Fall ist es eindeutig, $\mathrm{da} B \mathrm{~A}$ links von $\mathrm{B}$ und $\mathrm{B}$ rechts von $\mathrm{A}$ ist. Der Betrachter kann die Ballons auch beobachten, während er auf der Seite liegt, was die Abbildung $3 \mathrm{~b}$ zeigt.

(a)

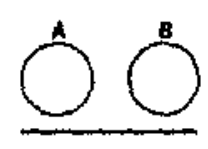<smiles>O=C1C2CC1C1C=CC2O1</smiles>

(d)
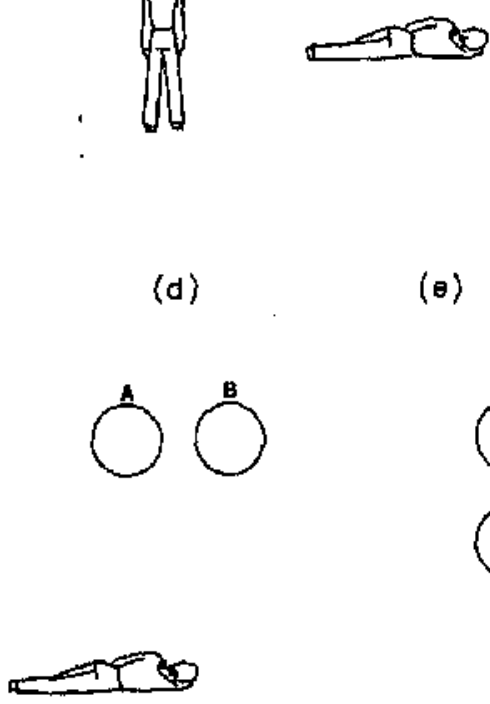

(घ)

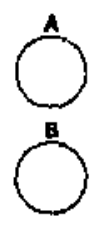

(b)
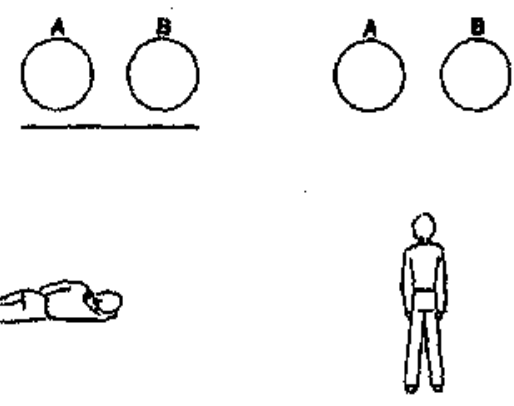

(1)
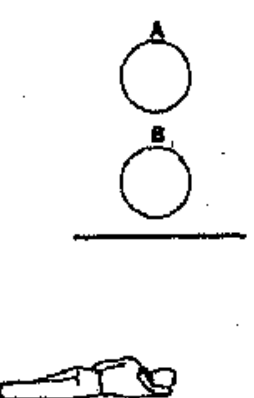

Abb. 3: Perzeptuell televante Informationen für den deiktischen Gebrauch von ,links* und, rechts'.

Diese Variation ist notwendig, um zu prüfen, ob es für den deiktischen Gebrauch von ,links' und, rechts ${ }^{4}$ von wesentlicher Redeutung ist, ob sich die Objekte parallel zur horizontalen Achse der Augen befinden oder nicht. In der Abbildung $3 \mathrm{~b}$ hat der Betrachter die Linie B-A parallel zur vertikalen Achse seiner Retinae. Einige Übungen auf dem 
Sofa haben mich davon überzeugt, daß es in solchen Fällen weniger angemessen ist zu sagen, daß $B$ rechts von $A$ ist, aber es ist wahrscheinlich immer noch möglich. Demgegenüber wäre es z. B. sicherlich nicht möglich zu sagen, daß sich $B$ in einem solchen Fall über $A$ befindet.

Aber eliminieren wir die visuellen Rahmenbedingungen, $\mathrm{d}$.h. den Horizont. Wenn die Ballons in einem ansonsten leeren Ganzfeld hängen und wenn der Betrachter wie in der Abbildung $3 c$ aufrecht steht, dann ist $A$ wiederum eindeutig links von $B$, und $B$ ist rechts von $A$. Visuelle Rahmenbedingungen sind für die Orientierung offensichtlich nicht obligatorisch, wenn es um die deiktische Zuweisung von, links' und ,rechts geht. Aber der Betrachter verfügt in diesem Fall über zwei weitere Informationsquellen. Die Objekte befinden sich parallel zar horizontalen Achse der Retinae, und über das Vestibularsystem weiB der Betrachter, daß die Anordnung orthogonal zur Richtung der Schwerkraft ist. Wir wollen dem Betrachter die erste Informationsquelle nehmen und uns vorstellen, $\mathrm{da} B$ er wie in der Abbildung $3 \mathrm{~d}$ auf der Seite liegt. Befindet sich in diesem Fall $\mathrm{A}$ immer noch links von B? Intuitionen stehen auf schwankenden Füßen, aber einige weitere Úbungen der Wahrnehmung einer weißen Wand yom Sofa aus haben mich davon überzeugt, đaß es unter diesen Umständen ein wenig merkwürdig ist zu sagen, daB A links von B ist, während es wahrscheinlich möglich ist zu sagen, daß sich $A$ unter B befindet.

$\mathrm{Um}$ zu überprüfen, welche Informationsquelle die wichtigere ist, Schwerkraft (die dem Betrachter etwas über die, objektive' horizontale Lage mitteilt) oder retinale Achse, muß man den in der Abbildung $3 e$ gegen den in der Abbildung 3d wiedergegebenen Fall testen. Bezüglich der Abbildung 3e informiert das Vestibularsystem den Betrachter darüber, daß sich die Ballons nicht auf einer horizontalen Ebene befinden, obwohl sie immer noch parallel zur horizontalen Ächse der Retinae sind. Ich glaube, daß man in diesem Fall sagen könnte, ,B ist rechts ven $A^{*}$, was darauf schließen ließe, daß die retinale gegenüber der vestibularen Information dominiert. Wenn jedoch die vestibulare Information durch einen visuellen Orientierungsrahmen unterstützt wird, wie z. B. durch den in der Abbildung $3 \mathrm{f}$ angedeuteten Horizont, dann scheint es kaum mehr möglich zu sein, daß man sagt, $B$ ist rechts von A. Der Ballon B befindet sich in diesem. Fall unter A.

Nicht alle Kombinationen der drei Arten perzeptueller Information können auf diese Weise untersucht werden; man würde beispielsweise ein Raumschiff benötigen, um die vestibulare Information ausschließen zu können. Unsere vorläufigen Überlegungen, die wir auf der Grundlage der Abbildung 3 angestellt haben, scheinen aber darauf zu verweisen, daß man im deiktischen System räumliche Relationen als ,links' oder ,rechts' ausdrücken kann, wenn bei mindestens zwei der drei Informationsquellen Übereinstimmung hinsichtlich der hori- 
zontalen Richtung festgestellt werden kann. Wenn zwei der drei Quellen eine vertikale Anordnung der Objekte andeuten, wie in Abbildung 3f, kann man ,über oder ,unter benutzen. Es sieht danach aus, als wäre keine der Informationsquellen eine notwendige Bedingung für den deiktischen Gebrauch der Ausdrücke, ,links ${ }^{6}$ und ,rechtss'. Oder, um es etwas genauer zu sagen, keine der Quellen muß notwendigerweise positive Information vermitteln; sie können immer durch die anderen kompensiert werden.

Wir wollen nun einige Bedingungen betrachten, die beim adäquaten Gebrauch des intrinsischen Systems erfüllt sein müssen. Es wurde bereits darauf hingewiesen, daß die Benutzung des intrinsischen Sy. stems eine Interpretation det Situation erfordert und zwar insbesonderc hinsichtlich der Art der beteiligten Objekte. Um auszudrücken, daß B rechts von $A$ ist, muß man sich vorher vergewissert haben, daß das Bezugsobjekt $\mathrm{A}$ eine intrinsische rechte Seite hat. Aber das ist nicht per se perzeptuell gegeben. Betrachten wir $z$.B. einige Objekte, bei denen wir davon ausgehen, da $B$ sie eine rechte und eine linke Seite haben: Personen, verschiedene Tiere, Autos, Kirchen, Stühle, Schreibtische etc. Diese Objekte verfügen durchaus nicht über eine gemeinsame Menge perzeptueller Merkmale. Zwischen ihnen besteht lediglich eine gewisse funktionale Beziehung, die uns durch vielfältige Erfahrungen bekannt ist. Die links/rechts-Struktur von Personen und (den meisten) Tieren ist wahrscheinlich eine Interpretation-durch-Analogie auf der Basis unserer eigenen links/rechts-Struktur. Die wichtigsten Siunesorgane befinden sich oben und vorn, und in dieser Weise orientiert sich det Organismus auch in seiner Umgebung. Auf dieser Grundlage schließen wir darauf, was links und rechts ist. Die links/rechts-Zuweisung bei Autos, Kirchen, Stühlen und Schreibtischen bestimmt sich aus der typischen räumlichen Orientierung, die Personen einnehmen, wenn sie sie benutzen. Das kann zu interessanten Komplikationen führen. Die Abbildung 4 (S. 196) zeigt einen Schreibtisch und einen Stuhl, und für jeden der beiden Gegenstände wurde vermerkt, was aus. intrinsischer Perspektive jeweils links, vorn und rechts ist.

Man sieht, daß für den Stuhl ,links', ,vorn', und ,rechts dieselben räumlichen Beziehungen ausdrücken, wie sie für Personen gelten. Für den Schreibtisch aber gilt, daß ,links' und , rechts ${ }^{4}$ hinsichtlich dessen, was ,vorn' ist, die Rollen vertauscht haben. Dies ist nur verständlich, wenn man die kanonische Position beachtet, die eine Person einnimmt, wenn sie einen Schreibtisch mit einem Stuhl benutzt. Für beide Gegenstände befindet sich das rechts, was sich rechts von der Person befindet und das links, was sich links von der Person befindet. Für den Stuhl ist ,vorn' die Richtung, in die die Person blickt, beim Schreibtisch aber ist die Seite die vordere, auf die die Person blickt. Wir werden diesen letzten Punkt noch im nächsten Abschnitt ausführlicher diskutieren. 


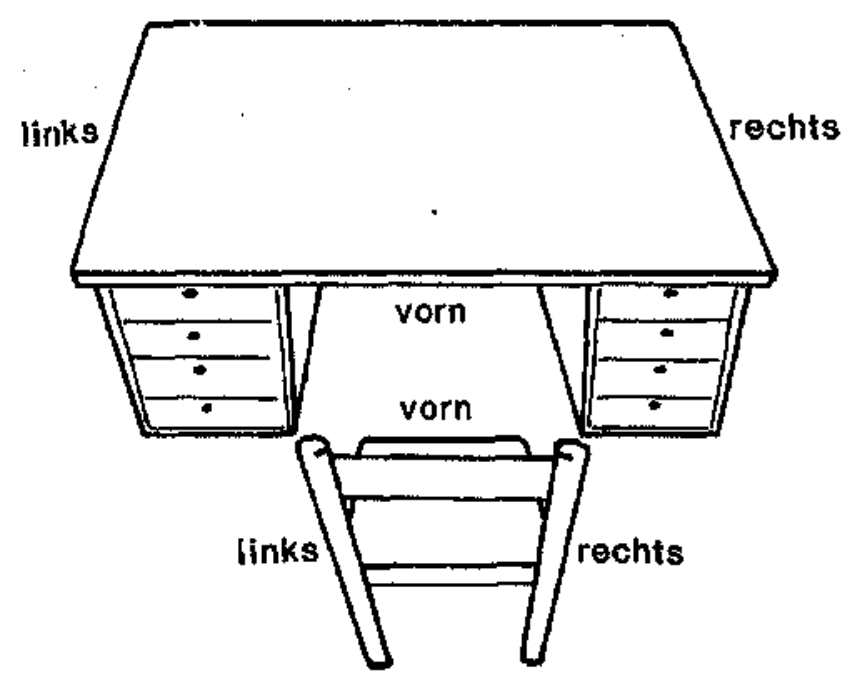

Abb.4: Unterschiede beim intrinsischen Gebrauch der Ausdrücke ,links', ,vorn' und ,rechts' beì einem Stuhl und einem Schreibtisch.

Eine sehr eingehende Behandlung dieser Aspekte des intrinsischen Systems enthält das Buch von Miller \& Johnson-Laird (1976).

Bisher konnten wir feststellen, da $B$ das intrinsische System unabhängig von der Perspektive des Sprechers ist; es beruht in erster Linie auf dem Wissen des Sprechers, das deiktische System dagegen auf perzeptuellen Daten. Wir werden in Kürze einen kleinen, aber interessanten Zusatz dahingehend machen müssen, da $B$ auch vor Anwendung des intrinsischen Systems bestimmte perspektivische Bedingungen erfüllt sein müssen. Davon wird am Ende des nächsten Abschnittes die Rede sein.

4. Deiktischer und intrinsischer Gebrauch der Ausdriucke, vor' und ,hinter'

Auch auf die Dimension, für die die Ausdrücke ,vor und ,hinter verwendet werden, kann man sowohl aus deiktischer als auch intrinsischer Perspektive verweisen. Folglich entstehen Probleme, die denen seht ähnlich sind, die wir bereits hinsichtlich der links/rechts-Dimension diskutiert haben. Allerdings sind die potentiellen Schwierigkeiten, die mit den Ausdrücken, vor' und ,hinten' verbunden sind, normalerweise weniger bewußt als dies für die Ausdrücke, links' und ,rechts ${ }^{2}$ gilt. Der wichtigste Grund dafür dürfte sein, $\mathrm{da} B$ die vor/hinter-Dimension funktional und morphologisch asymmetrisch ist, während sich links/ rechts diesbezüglich weitestgehend symmetrisch verhalten. Die Bedeutung der Ausdrücke ,vor' und, hinter' steht in enger Beziehung zu der aus biologischer Sicht eminent wichtigen Unterscheidung dessen, was 
sich, näher zu mir" oder, weiter von mir" befindet sowie zu der Unterscheidung der vorderen und hinteren Körperfläche (diese Überlegungen werden in Clark (1973) ausführlich diskutiert). Hinsichtlich der Unterscheidung von links und rechts lassen sich keine Gesichtspunkte von vergleichbarer funktionaler oder morphologischer Bedeutung finden.

Betrachten wir zunächst die Eigenschaften der Umkehrbarkeit und Transitivität für die Ausdrücke, vor' und ,hinter', Die Abbildung 5a zeigt zwei sich nicht bewegende Ballons und einen Betrachter aus der Vogelperspektive. Die beiden Gegenstände und die Person befinden sich ungefähr in einer Linie. Wir wollen bei diesem und bei allen folgenden Fällen davon ausgehen, daß sich alle Objekte in sicht des Betrachters befinden.
(a)
(b)
(c)
(d)<smiles>[C]1CCCCC1</smiles>
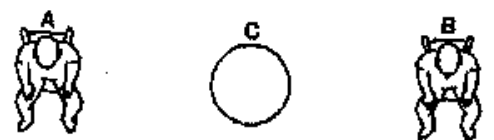
$\overbrace{}^{8}$
$0_{0}^{1} 3$<smiles>C1CCCCC1</smiles>

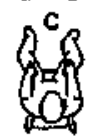
V
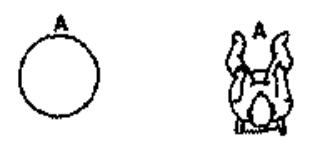
$\checkmark$
5 (c) 5 (d)
A lat vor $B \quad$ A tst vor $B$
$B$ ist vor $C \quad B$ ist vor $C$
A lat vor C A ist hinter C

Abb. 5: Umkehrbarkeit und Transitivität bei deiktischem und intrinsischem Gebrauch der Ausdrücke, vor" und ,hinter".

Unter diesen Bedingungen ist $B$ vor $A$, und $A$ befindet sich hinter $B$. Es scheint, daß die Beziehungen unter diesen Umständen umkehrbar sind, und es ist mir nicht gelungen, einen Fall zu finden, für den unter deiktischer Perspektive keine Umkehrbarkeit gilt, wenn sich die Position des Betrachters dabei nicht verändert. Die Abbildung $5 b$ kann intrinsisch interpretiert werden. In diesem Fall ist die Position des Betrachters irrelevant, und sowohl $A$ als auch $B$ haben eine jeweils 
eigene Orientierung. Es ist eindeutig, daß B vor $A$ und $A$ vor $B$ sitzt, hier ist die Beziehung also nicht umkehrbar. Man könnte natürlich $A$ und $B$ so plazieren, dabs sich $A$ vor $B$ und $B$ hinter $A$ befinden, aber hier geh $\downarrow$ es darum, daß ,vor" und ,hinter" im intrinsischen System nicht notwendigerweise in einer umkehrbaten Beziehung zueinander stehen, während eben dies offensichtlich für das deiktische System gilt.

Bevor wir uns der Transitivität zuwenden, muß eine wichtige Beobachtung von Hill (1982) aufgegriffen werden. Hill hat darauf hingewiesen, daß nicht alle Sprachen die Ausdrücke, vor ${ }^{c}$ und ,hinter in der gleichen Weise verwenden, wie wir das bezüglich der Abbildung 5 a diskutiert haben. Die afro-asiatische Sprache Hausa erfordert, daß der Betrachter in dieser Situation sagt, daß sich A vor B befindet, zumindest wenn $A$ deutlich sichtbar ist. Hill nennt Hausa eine, aligning language'. Es scheint, als ob die Orientierung von B gegenüber A dieselbe ist wie die Orientierung des Betrachters gegenüber A; A befindet sich in der gleichen Weise vor B wie es sich vor dem Betrachter befindet. Deutsch, Englisch, Niederländisch und viele andere Sprachen verhalten sich anders, es sind ,facing languages". In diesen Sprachen ist es, als ob B den Betrachter ansieht, so daB sich A hinter B befindet. Dennoch trifft wahrscheinlich $\mathrm{zu}, \mathrm{da} B$ in einer , aligning language' die Eigenschaft der Umkehrbarkeit für das deiktische System gilt, so wie auch in einer, facing language'.

Das deiktische System ist auch für ,vor" und ,hinter ${ }^{4}$ transitiv. Dies zeigt die Abbildung 5c. A ist vor $B, B$ ist vor $C$, und $A$ ist vor C. In diesem Zusammenhang habe ich keinen Fall gefunden, bei dem für das deiktische System die Bedingung der Transitivität nicht gilt. Es ist dagegen einfach, Beispiele dafür zu finden, daß bei der Verwendung des intrinsischen Systems die räumlichen Beziehungen nicht transitiv sind. Ein solches Beispiel zeigt die Abbildung $5 \mathrm{~d}$, die sich analog zur Abbildung $2 \mathrm{~d}$ verhält. $\mathrm{A}$ ist vor $\mathrm{B}, \mathrm{B}$ ist vor $\mathrm{C}$, aber $\mathrm{A}$ ist hinter $\mathrm{C}$. Die Tatsache, daß die Ausdrücke, vor' und ,hinter ${ }^{6}$ im intrinsischen Siystem nicht vollständig umkehrbar und transitiv sind, könnte die Verwendung dieser Ausdiücke in der gleichen Weise erschweren, wie wir es bereits bezüglich der intrinsisch verwendeten Ausdrücke ,links" und ,rechts" beobachtet haben.

Wenn wir jetzt dazu übergehen, einige perzeptuelle Bedingungen des deiktischen Gebrauchs der Ausdrücke, vor' und ,hinter“ zu diskutieren, müssen wir zunächst etwas korrigieren, was in der bisherigen Literatur in bemerkenswerter Weise durcheinandergeraten ist. Weiter oben haben wir die Objekte A und B der Abbildung 5a als statische Objekte aufgefaßt. Hill (1982) hat festgestellt, daß die deiktische Verwendung der Ausdrücke, vor' und, hinter davon abhängt, ob $\mathrm{A}$ und B statische oder dynamische Objekte sind. Diese Überlegung wird durch die Abbildung 6 illustriert. 
(a)

(b)

(c)<smiles>C1CCCCC1</smiles><smiles>[C+]1CCCCC1</smiles>

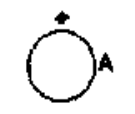<smiles>[C]1CCCCC1</smiles><smiles>[C]1CCCCC1</smiles><smiles>[C]1CCCCC1</smiles><smiles>[C]1CCCCC1</smiles><smiles>[C]1CCCCC1</smiles><smiles>[C]1CCCCC1</smiles><smiles>CC1CCCCC1</smiles>

$\mathrm{V}$ $\checkmark$ (d)

(e)

Abb. 6: Deiktische und intrinsische Perspektive in statischen und dynamischen Situationen

Abbildung $6 \mathrm{a}$ ist eine Kopie der Abbildung 5a. Sie zeigt den Betrachter und die statischen Objekte $A$ und B. Man beachte, daß sich B aus der Perspektive des Betrachters vor A befindet. Abbildung $6 \mathrm{~b}$ unterscheidet sich von $6 a$ nur darin, daß Pfeile anzeigen, daß sich die Objekte vom Betrachter wegbewegen. Hill hat festgestellt, daB sich A in diesem Fall vor $B$ befindet und $B$ hinter $A$ ist. Diese Beobachtung ist zwar völlig korrekt, nicht aber deren Interpretation.

Betrachten wir zunächst Abbildung 6c, die sich wiederum nur durch die hinzugefügten Pfeile von der Abbildung 6a unterscheidet, welche diesmal jedoch die entgegengesetzte Bewegungsrichtung anzeigen. Nun ist die Situation die gleiche wie in der Abbildung 6a: B ist vor $A$, und $A$ ist hinter $B$. Damit ist es nicht der dynamische Charakter der Situation, der für die Umkehrung von, vor' und, hinter' verantwortlich zu machen ist, wie Hill angenommen hat. Entscheidend dafür ist vielmehr die Bewegungsrichtung. Noch wichtiger aber ist, $\mathrm{daß}$ die Position des Betrachters irrelevant ist. Obwohl der Betrachter die Bewegung der Objekte $\mathrm{A}$ and $\mathrm{B}$ aus der Perspektive der Abbildung $6 \mathrm{~b}$ oder $6 \mathrm{~d}$ beobachtet, $\mathrm{A}$ befindet sich immer vor B und B immer hinter A. Wenn aber die Verwendung der Ausdrücke, vor" und ,hinter" unabhängig von der Position des Betrachters ist, dann können wir ziemlich sicher sein, daß wir uns hier innerhalb des intrinsischen Systems bewegen und nicht innerhalb des deiktischen wie Hill (und mit ihm Ehrich, 1985) angenommen haben. Objekte, die aus intrinsischer Sicht über keine Vorderseite verfügen, erwerben durch ihre Bewegung eine solche Vorderseite; sie ist die am meisten nach vorn gerichtete Seite des sich bewegenden Objekts. Wir können daher annehmen, daß die deiktische Eigenschaft der Umakehrbarkeit nicht ohne weiteres für bewegte Objekte gilt. Abbildung 6e zeigt, da $B$ diese Annahme richtig ist. In dieser Abbildung bewegt sich $A$ auf $B$ zu, und $B$ bewegt sich in 
entgegengesetzter Richtung auf $A$ zu. Bezogen auf diese Situation kann man ohne Zweifel sagen, daB B vor A und A vor B ist.

Die Bedeutung von Hills Beobachtung besteht darin, $\mathrm{daß}$ es unter bestimmten Bedingungen wie etwa der Abbildung $6 \mathrm{~b}$ schwierig werden kann, das deiktische System zu benutzen; der Sprachbenutzer sieht sich fast dazu gezwungen, zum intrinsischen System überzugehen. Ehrich (1985) hat eine ähnliche Beobachtung gemacht bezüglich einer Situation, die mit der der Abbildung $5 b$ vergleichbar ist. Es ist außerordentlich schwierig in einem solchen Fall das deiktische System zu benutzen. Wäre der Betrachter hinsichtlich der Objekte A und B in der gleichen Position wie der Betrachter der Abbildung 5a, dann wäre es sehr schwierig zu sagen "A ist hinter $\mathrm{B}^{\prime \prime}$, auch wenn dies im deiktischen System korrekt wäre. Mit anderen Worten, wenn ein Bezugsobjekt entweder ohnehin oder dadurch, daß es sich bewegt, über eine intrinsische Vorderseite verfügt, besteht eine sehr starke Tendenz, fürt , vor' und ,hinter' das intrinsische System zu verwenden. Darin unterscheiden sich diese Ausdrücke deutlich von den Ausdrücken ,links' und ,rechts': die Situation der Abbildung 1b erlaubt für letztere gleichermaßen einen deiktischen oder intrinsischen Gebrauch. Ein solches Gleichgewicht gilt nicht für die Situation der Abbildung 5b.

Wenn wir jetzt auf die Verwendung des deiktischen Systems zurückkommen und uns fragen, welche Situations-Bedingungen erfüllt sein müssen, um zu sagen, da $B$ sich $A$ vor $B$ befindet, müssen wir die allgemeinen Informationsquellen der Tiefenwahrnehmung in Betracht ziehen. Handbücher der visuellen Wahrnehmung wie z. B. Kaufman (1974) geben einen ausführlichen Ưberblick über solche Informationsquellen, worauf hier nicht eingegangen zu werden braucht. Der Betrachter von Abbildung 6a kann daraus schließen, daB sich B vor $A$. befindet, entweder indem er die Okklusionsbeziehungen zwischen beiden Objekten betrachtet, durch Stereopsis oder: Bewegungsparallaxe, durch einen Vergleich des durch A und B beanspruchten Blickwitikels etc. Für den Gebrauch der deiktischen Terminologie ist es nicht völlig irrelevant, welche Informationsquelle benutzt wird. Hills (1982) Beobachtung, daß sich für einen Sprecher des Hausa B in der Situation der Abbildung 6a hinter $\mathrm{A}$ befindet, ist nur dann zutreffend, wenn sowohl A und $B$ deutlich sichtbar sind. Wenn A durch B verdeckt wird, der Sprecher aber weiß, daß sich $A$ auf der anderen Seite von B befindet, dann wird man sagen, daß sich $A$,hinter' $B$ befindet.

Okklusion scheint auch eine besondere Rolle bei der Verwendung der Ausdrücke, vor' und ,hinter' im Deutschen zu spielen. Das hat allerdings mit der Unterscheidung von horizontalen und vertikalen Anordnungen $\mathrm{zu}$ tun. Es sei daran erinnert, $\mathrm{da} B$ es hinsichtlich der deiktischen Verwendung von ,links ${ }^{l}$ und ,rechts' eindeutige Bedingungen der Horizontalität für einen auf einer Ebene stehenden Betrachter 
der Objekte A/B gibt. Stellen wir uns nun vor, daB A, B und der Betrachter sich in der Abbildung $6 \mathrm{a}$ vertikal zueinander befinden, wobei $\mathrm{A}$ die höchste Position einnimmt und der Betrachter von der niedrigsten Position aus nach oben schaut. Der Betrachter könnte dann sagen, daß sich A über B befindet. Dazu wird im nächsten Abschnitt mehr gesagt werden. An dieser Stelle wollen wir überlegen, ob der Betrachter in dieser Situation auch sagen könnte „B ist vor $A^{\text {" }}$ oder "A ist hinter $B$ ". Diese Frage scheint man dann bejahen zu können, wenn A durch B teilweise oder ganz verdeckt wird. Man kann in den Himmel schauen und sagen "die Sonne ist hinter den Wolken" oder "vor der Sonne sind Wolken", aber das setzt yoraus, daß die Wolken die Sonne ganz oder zum Teil verdecken. Es wäre tuisăchlich falsch $z \mathfrak{z}$ sagen, daß die Sonne hinter den Wolken ist, wenn sie von ihnen nicht verdeckt wird. Bei der Verwendung des Ausdrucks, über‘ gibt es keine solche Beschränkung. Man kann auch dann sagen, daß die Sonne über den Wolken steht, wenn es keine Abdeckung gibt; es bedeutet einfach, daß sich die Sonne in einer höheren Position als die Wolken befindet (vgl. den folgenden Abschnitt). Wenn es korrekt ist zu sagen, daß die deiktische Verwendung der Ausdrücke, vor $/$,hinter genauso wie die deiktische Verwendung von, links" und, rechts' eine in etwa horizontale Anordnung von Betrachter und Objekten erfordert, sollte man die Möglichkeit in Betracht ziehen, daß bei diesen Fällen yon Okklusion in gewisser Weise das intrinsische System beteiligt ist. Es ist nicht ausgeschlossen, daB Wolken mit einer intrinsischen Vorderseite wahrgenommen werden (als der dem Betrachter zugewandten Seite) oder daB, allgemeiner, ein verdeckendes Objekt, welches zum Bezugsrahmen für das verdeckte Objekt wird, in der gleichen Weise wie ein bewegendes Objekt eine intrinsische Vorderseite erwirbt. Diese Möglichkeit wird an dieser Stelle allerdings nicht weiter diskutiert werden.

Wir wollen nun zum normalen intrinsischen Gebrauch der Ausdrücke ,vor"/,hinter" übergehen sowie zu den ihn einschränkenden perzeptuellen Bedingungen. Die Abbildung 7a (S. 202) zeigt tinen Ballon der sich, vor ${ }^{6}$ dem Stuhl befindet, wenn man die intrinsische Orientierung des Stuhles in Rechnung stellt. Wie resistent ist der intrinsische Gebrauch gegenüber Informationen der deiktischen Orientierung? In der Abbildung $7 \mathrm{~b}$ ist der Stuhl umgekippt, aber auch der Ballon befindet sich an anderer Stelle, so daß dieselbe räumliche Beziehung zwischen Stuhl und Ballon wie in der Abbildung 7a erhalten bleibt. In diesem Fall scheint es wirklich ausgeschlossen zu sein, die Situation durch die Äußerung "Der Ballon ist vor dem Stuhl“" zv. beschreiben, vielmehr befindet er sich, über" dem Stuhl. 


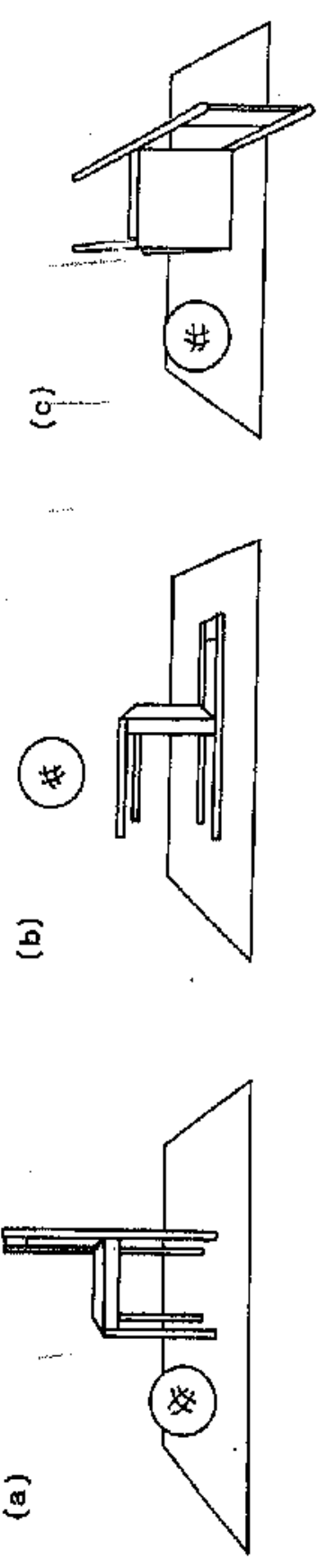

$\frac{8}{4} \ldots \ldots \ldots-\ldots$

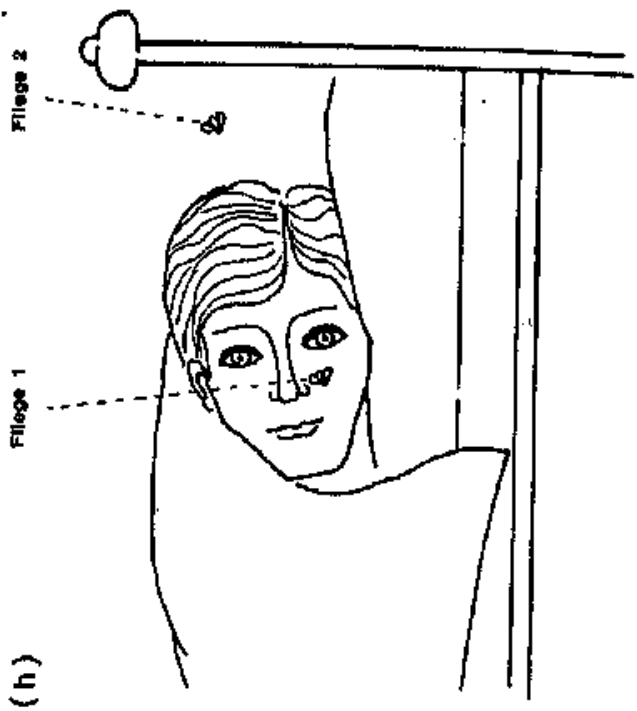

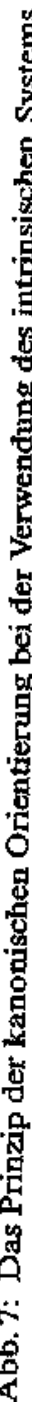


Wie läßt sich das verstehen? Offensichtlich ist'der intrinsische Gebrauch der Präposition ,vor abhängig von der ,kanonischen' oder normalen Position des Bezugsobjekts in Hinsicht auf den perzeptuellen Orientieruagsrahmen des Verweisobjekts. Bei Abweichungen von dieser kanonischen Position wird der perzeptuelle Orientierungsrahmen dominant, und das deiktische System wird benutzt. (Die deiktische Verwendung von, vor' wird im nächsten Abschnitt diskutiert werden.) Utberraschenderweise kann man allerdings beobachten, daß das intrinsische System immer noch auf eine Dimension angewendet werü?: kann, die bei einer Rotation des Bezugsobjekts erhalten bleibt. Ein Beispiel dafür zeigt die Abbildung 7c, wo der Stuhl cbenfalls eine nicht-kanonische Position einnimmt, wobei jedoch die Dimüusion der Vorder- und Rückseite hinsichtlich des perzeptuellen Orientierungsrahmens erhalten bleibt. In diesem Fall ist es ziemlich normal, wenn man sagt, daß sich der Ballon vor dem Stuhl befindet. Die rotierte Dimension bezieht sich hier auf das Oben und Unten des Stuhles, und Abbildung $7 \mathrm{~d}$ zeigt, daß man in diesem Fall kaum sagen kann „Der Ballon befindet sich über dem Stuhl“", was man durchaus bei der Abbildung $7 \mathrm{e}$ sagen kann, bei der die gleiche räumliche Beziehung zwischen Stuhl und Ballon gilt.

Der Schluß scheint daher gerechtfertigt zu sein, daß der intrinsische Gebrauch von ,vor $\%$, hinter" und, über \%, unter" nur für eine Dimension des Bezugsobjekts möglich ist, die nicht bezüglich des perzeptuellen Orientierungsrahmens des Verweisobjekts (falls es ein solches gibt) rotiert wird.

Man sollte nun die Frage stellen, ob das gleiche für die links/ rechts-Dimension gilt. Abbildung $7 \mathrm{f}$ zeigt einen Fall, wo diese Dimension $\mathrm{zu}$ einer nicht-kanonischen Orientierung verändert wurde. Tatsächlich scheint es merkwürdig, wenn nicht sogar völlig unangemessen zu sein, wenn man sagt, daß sich der Ballon nun rechts vom Stuhl befindet. Bei der Abbildung $7 \mathrm{~g}$ ist die rechts/links-Dimension des Stuhles bei der Rotation erhalten geblieben, und es scheint ohne weiteres möglich zu sein, wenn man in diesem Fall sagt, daß der Ballon rechts vom Stuhl ist. Die Schlußfolgerung kann nun für alle drei Dimensionen in der folgenden. Weise verallgemeinert werden:

Prinzip der kanonischen Orientierung. Um mit dem intrinsischen System auf die intrinsische Dimension eines Bezugsobjektes zu verwsisen, $\mathrm{muB}$ sich diese Dimension hinsichtlich des perzeptuellen Orientierungsrahmens des Verweisobjekts in kanonischer Position befinden.

Der perzeptuelle Orientierungsrahmen wird gewöhnlich mit der wahrgenommenen Vertikalen übereinstimmen, die wiederum abhängt 
von den visuellen Rahmenbedingungen, den Informationen vestibularer oder retinaler Orientierung. Dies trifft zu für alle diskutierten Beispiele der Abbildungen 7a bis 7g. Weiterhin werden die perzeptuellen Rahmenbedingungen oder der Hintergrund des Verweisobjektes gewöhnlich auch die perzeptuellen Rahmenbedingungen oder der Hintergrund des Bezugsobjekts sein. Auch das gilt für alle Fälle der Abbildungen $7 \mathrm{a}$ bis $7 \mathrm{~g}$.

Es ist jedoch nicht unmöglich, Ausnahmen zu konstruieren, d.h. Fälle, wo der wahrgenommene Hintergrund als Rahmen für das Verweisobjekt nicht mit dem Hintergrund als Rahmen für das Bezugsobjekt übereinstimmt und wo deren Orientierung verschieden ist. Solch êtine Ausnahme zeigt die Abbildung 7h, wo auf dem Gesicht einer Person, die wir Peter nennen wollen, eine Fliege sitzt und sich zwei weitere Fliegen in der Nähe befinden. Der perzeptuelle Orientierungsrahmen der ersten Fliege ist das Gesicht von Peter, während der perzeptuelle Orientierungsrahmen für das Gesicht von Peter seinerseits durch das Bett und die damit wahrgenommene Vertikale gebildet wird. Wenn man das Prinzip der kanonischen Orientierung strikt anwendet, kommt man zu der Vorhersage, da $\beta$ es angemessen ist zu sagen, "Links von Peters Nase befindet sich eine Fliege". Die intrinsische (horizontale) Dimension, die die Fliege zu Peters Nase in Beziehung setzt, ist in einer kanonischen Position hinsichtlich des perzeptuellen Orientierungsrahmens, der aus Peters Gesicht besteht (sowohl für die Fliege als auch für die Nase).

Die zweite Fliege ist so nahe an Peters Kopf, daß das Gesicht auch für diese Fliege als perzeptueller Orientierungsrahmen dienen kann. Dementsprechend kann man dem Prinzip der kanonischen Orientierung folgend sagen , $\mathrm{Da}$ ist eine Fliege über Peters $\mathrm{Kopf}{ }^{*}$. In diesem Fall bildet Peters Kopf den perzeptuellen Orientierungsrahmen für das Verweisobjekt (die zweite Fliege). Die intrinsische (vertikale) Dimension, die die Fliege zu Peters Kopf in Beziehung setzt, ist hinsichtlich dieses Orientierungsrahmens in kanonischer Position. Aber an dieser Stelle sollte angemerkt werden, daß das Bett den perzeptuellen Rahmen für das Bezugsobjekt (Peters Kopf) bilcet, das in anderer Weise orientiert ist. Es ist daher von wesentlicher Bedeutung, daß das Prinzip der kanonischen Orientierung in bezug auf den perzeptuellen Orientierungsrahmen des Verweisobjekts formuliert wird. Für die erste und zweite Fliege bildet Peters Kopf den perzeptuellen Orientierungsrahmen. In Abbildung 7d aber ist der perzeptuelle Orientierungsrahmen des Ballons nicht der Stuhl, sondern die Matte auf dem Fußboden. Deshalb ist der Ballon nicht über dem Stuhl, während sich die zweite Fliege über Peters Kopf befindet. Mit anderen Worten, das Bezugsobjekt kann, muß aber nicht den Orientierungsrahmen des Verweisobjekts bilden. Die dafür entscheidenden Bedingungen sind vermutlich gestal- 
tartig und beziehen sich auf Vorder- und Hintergrund sowie GröBenrelationen. Sie sollen hier nicht weiter untersucht werden.

Die dritte Fliege schließlich befindet sich in ziemlich großer Entfernung von Peters Kopf. In dieser Situation wird Peters Kopf gewöhnlich nicht ihren perzeptuellen Orientierungsrahmen bilden, dennoch hat die Szene als solche eine eindeutige vertikale Orientierung. Diese Orientierung stimmt nicht überein mit der intrinsischen vertikalen Dimension, die erforderlich ist, um zu sagen „Da ist eine Fliege über Peters Kopf". Korrekterweise verbietet das Prinzip diese letzte Äußerung bezogen auf eine Fliege, die dermaßen weit entfernt ist.

Es ist nicht schwierig, ähnliche Beispiele für die Dimension ,vor'/ ,hinter ${ }^{*}$ zu konstruieren, die der hauptsächliche Gegenstand dieses Abschnittes ist. Wenn Peter beispielsweise zur Decke aufschauen würde, und es befände sich eine Fliege (deiktisch) gerade über seiner Nase, könnte man ohne weiteres sagen "Da ist eine Fliege vor Peters Nase." Dies wäre für eine weiter entfernte Fliege der Vorhersage entsprechend nicht möglich. Das Prinzip der kanonischen Orientierung hat eine unerwartete, aber wichtige Folge für die Verwendung der Ausdrücke ,über' und ,unter', die im folgenden Abschnitt diskutiert werden soll.

\section{Deiktischer und intrinsischer Gebrauch der Ausdrücke ,über' und ,unter"}

Die letzte Dimension, die wir betrachten wollen, ist die vertikale. Sie unterscheidet sich in wesentlichen Beziehungen von den vorangegangenen zwei Dimensionen, sowohl hinsichtlich des deiktischen als auch des intrinsischen Gebrauchs. Wir wollen uns zunächst den deiktischen Gebrauch anschauen. Die erste Beobachtung, die man macht, besteht darin, daB der Punkt, von dem aus der Betrachter bzw. Sprecher die Szene sieht, für den deiktischen Gebrauch der Ausdrücke ,über' und ,unter" irrelevant ist. Es sei daran erinnert, daß wir den deik tischen Gebrauch dadurch definiert haben, daß räumliche Begriffe aus der Perspektive des Betrachters/Sprechers interpretiert werden. Welcher Aspekt der Perspektive spielt für đen deiktischen Gebrauch von, äber und ,unter" eine Rolle, wenn der Punkt, von dem aus der Betrachter eine Szene wahrnimmt, irrelevant ist? Es ist die vertikale Orientierung der Perspektive des Sprechers. Diese Tatsache verdeutlicht die Abbildung 8 (S. 206).

Nehmen wir an, daB der Betrachter, der sich in Position 1 der Abbildung $8 \mathrm{a}$ befindet, den Ballon $\mathrm{A}$ als ïber Ballon $\mathrm{B}$ wahrnimmt (und damit $\mathrm{B}$ als unter $\mathrm{A}$ ). Wenn der Betrachter nun Position 2 einnimmt und nach oben schaut, kann er immer noch sagen, daß $A$ über $B$ und $B$ unter $A$ ist. Das gleiche gilt, wenn der Betrachter zur 


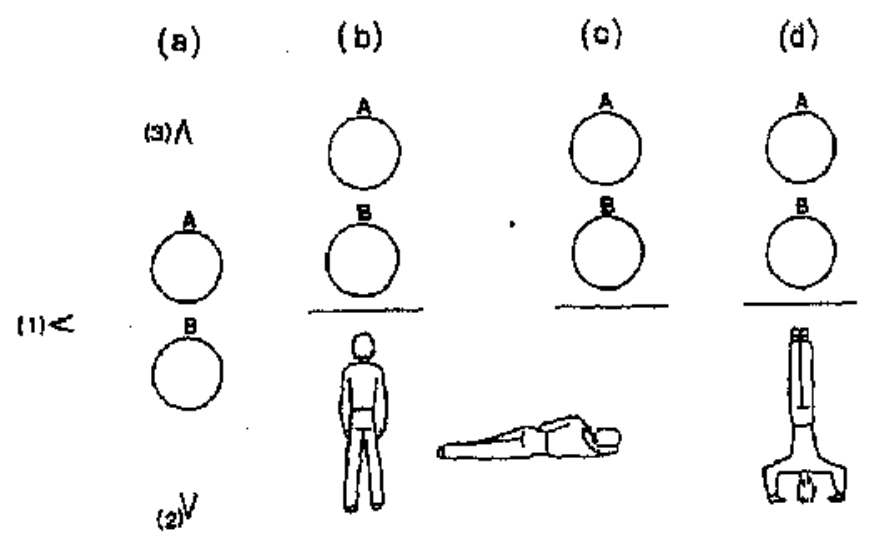

Abb.8: Einige Verwendungsmöglichkejten für ,über' und ,unter'.

Position 3 übergeht und auf die Ballons hinabschaut; auch von dieser Position aus befindet sich $\mathrm{A}$ über $\mathrm{B}$ und $\mathrm{B}$ unter $\mathrm{A}$. Das zeigt, daB die Position des Sprechers in bezug auf die Szene unwesentlich ist. Auch die Orientierung des Körpers vom Betrachter bzw. Sprecher spielt keine Rolle. Er nimmt wahr, daß sich A über B befindet, wenn er wie in Abbildung $8 \mathrm{~b}$ aufrecht steht. Daran ändert sich auch dann nichts, wenn der Betrachter sich wie in Abbildung $8 \mathrm{c}$ hinlegt.

Was wesentlich ist, ist die wahrgenommene Vertikalität und zwar in der gleichen Weise, in der die wahrgenommene Horizontalität wesentlich für die deiktische Verwendung von, links ${ }^{4}$ und ,rechts ${ }^{\star}$ war. Und auch die benutzten Informationsquellen sind analog. Als Grundlage dafür, daß der Betrachter die Szene als vertikal organisiert wahrnimmt, kommen in Betracht: (1) ihre Beziebung zur vertikalen Achse der Retina, (2) ihre Orientierung hinsichtlich bestimmter visueller Rahmenbedingungen (Horizont o. ä.) und (3) ihre Beziehung zur Vertikaliiatb die das Vestibularsystem vermittelt. Die erste Informationsquelle spielt eine Rolle in der Abbildung 8b, nicht aber in $8 \mathrm{c}$ (die identisch ist mit $3 \mathrm{f}$ ) oder $3 \mathrm{e}$. Die zweite Informationsquelle wird benutzt in Abbildung $8 \mathrm{~b}$ und $8 \mathrm{c}(=3 \mathrm{f})$, nicht aber in den Abbildungen $3 \mathrm{~d}$ und 3e. Die vestibulare. Information schlieblich ist entscheidend in den Abbildungen $8 \mathrm{~b}, 8 \mathrm{c}(=3 \mathrm{f})$ und 3e, nicht aber in Abbildung 3d. Keine dieser Informationsquellen ist unbedingt notwendig; der Eindruck der Vertikalität kann durch jede einzeln hervorgerufen werden. Die Situation ist sehr ähnlich zu der, die wir in Verbindung mit Abbildung 3 diskutiert haben; wir wollen an dieser Stelle nicht weiter darauf eingehen.

Im deiktischen System gelten die üblichen Eigenschaften der Umkehrbarkeit und Transitivität für, über ${ }^{c}$ und ,unter ${ }^{*}$. Daß es sich diesbezüglich vollständig regelhaft verhält, ist nicht einfach trivial, wenn man 
bedenkt, daß der Punkt, von dem aus der Betrachter/Sprecher die Szene sieht, in diesem deiktischen System irrelevant ist.

Gibt es überhaupt eine intrinsische Verwendung von, ̈̈ber" und ,unter"? In der Literatur wird diese Frage einmütig bejaht. Wenn ein Objekt wie eine Person, ein Auto oder ein Haus ein intrinsisches Oben hat, dann kann etwas ,uber diesem Objekt sein. Wenn ein Objekt über eine intrinsische Unterseite verfügt so wie ein Stuhl, ein Flugzeug oder ein Affe, dann kann in der gleichen Weise etwas, unter ihm sein. Dennoch gibt es gute Gründe, um zu bezweifeln, ob die Ausdrücke ,über'/,unter' auf eine Art intrinsich benutzt werden können, die vergleichbar ist mit der intrinsischen Verwendung von, links $\%$,rechts' oder ,vor'/,hintert, und wir werden zeigen, daß dies eine ganz natürtiche Konsequenz des oben diskutierten Prinzips der kanonischen Orientierung ist.

Die Schwierigkeiten bei der intrinsischen Verwendung von ,über' und ,unter' werden sofort offensichtlich, wenn man versucht, Fälle zu konstruieren, bei denen die Bedingungen der Umkehrbarkeit und Transitivität verletzt werden. Dies scheint unmöglich zu sein. Abbildung 9 a zeigt zwei Stühle in einer vertikalen Anordnung. Ohne Frage befindet sich Stuhl B unter Stuhl A. Es wäre jedoch völlig unangemessen zu sagen, „Stuhl A ist unter Stuhl B“. Trotz der Ähnlichkeit zu der in der Abbildung 5 b dargestellten Situation, wo die Umkehrbarkeit von ,vor\%, hinter" verletzt worden war, gilt hier strikte Umkehrbarkeit. Daran ändert sich auch nichts, wenn man die Situation wie in der Abbildung $9 \mathrm{~b}$ symmetrischer macht. In diesem Fall kann man weder sagen, daB $A$ unter $B$, noch $\operatorname{daB} B$ unter $A$ ist.

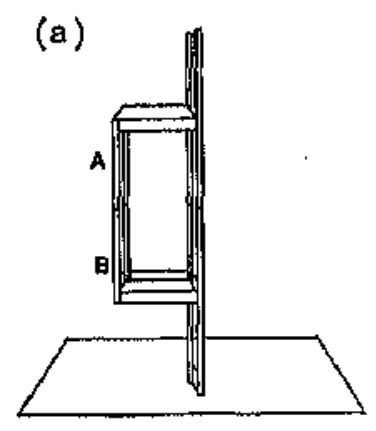

(b)

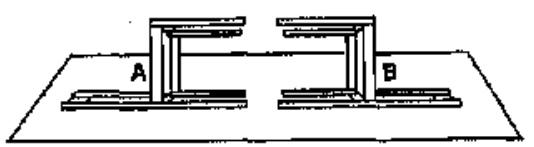

Abb. 9: ,Über', ,unter' und das Prinzip der kanonischen Orientierung.

Der Grund, warum Umkehrbarkeit (und in diesem Fall Transitivität) in diesen oder ähnlichen Fällen nich t verletzt werden können, besteht darin, daß mindestens eines der beiden Objekte in bezug auf die perzeptuellen Rahmenbedingungen, d.h. hinsichtlich dessen, was 
als vertikal wahrgenommen wird, nicht seine normale Position einnimmt. In beiden Situationen, 9a und 9b, wird das Prinzip der kanonischen Orientierung verletzt. Abbildung $9 \mathrm{a}$ zeigt Stuhl A, nicht aber Stuhl B in kanonischer Position. Daher ist es unmöglich, ,unter B“ intrinsisch $\mathrm{zu}$ verwenden. In A bbildung $9 \mathrm{~b}$ sind beide Stühle in nicht. kanonischer Position. Man kann daher weder sagen, "B ist unter A“ noch „A ist unter $B^{\star 4}$. Offensichtlich gibt es keine Lösung, bei der sich beide Stühle bezüglich eines gemeinsamen perzeptuellen Orientierungsrahmens in kanonischer Position befinden.

Damit aber hat das Prinzip weitreichende Konsequenzen für alle Fälle, bei denen der perzeptuelle Orientierungsrahmen für zwei Objekte derselbe ist: intrinsischer Gebrauch von, über/,unter ${ }^{*}$ ist genau in den Fällen möglich, in denen auf Grund des deiktischen Systems dieselben Ausdrücke verwendet würden. Mit anderen Worten, sobald eine intrinsische Verwendung dieser Ausdrücke mit ihrer deiktischen Verwendung in Konflikt geraten sollte, wird das intrinsische System durch das Prinzip der kanonischen Orientierung blockiert. Man könnte auch sagen, $\mathrm{daB}$ es keinen genuinen intrinsischen Gebrauch von ,über" und ,unter" unter diesen ziemlich allgemeinen Bedingungen gibt. Das unterscheidet sich deutlich von dem, was wir hinsichtlich des intrinsischen Gebrauchs von ,links'/,rechts" sowie ,vor $\%$,hinter" beobachten konnten.

Bedeutet das auch, daß der intrinsische Gebrauch von ,über"/ ,unter" ganz allgemein unmöglich ist? Nein, das Prinzip der kanonischen Orientierung bietet ein Schlupfloch, das wir in Verbindung mit der Abbildung $7 \mathrm{~h}$ diskutiert haben. Dort war es möglich zu sagen, da 3 die zweite Fliege über Peters Kopf ist. Wenn sich die erste Fliege zwischen Peters Augen setzen würde, könnte man in der gleichen Weise sagen, "Úber Peters Nase ist eine Fliege"; odẹr wenn sie auf Peters Oberlippe landen würde, könnte man durchaus sagen „Unter Peters Nase ist eine Fliege". Wie wir bereits festgestellt haben, bildet in solchen Fällen der Kopf von Peter den Orientierungshintergrund für die Wahrnehmung der Fliege; die Lage des Kopfes stimmt nicht überein mit der Lage des Hintergrundes, vor dem dieser Kopf wahrgenommen wird, und daher kann man ,über' und ,unter' intrinsisch verwenden. Dennoch ist es selbst in điesen Fällen nicht möglich, Bedingungen der Umkehrbarkeit und Transitivität zu verletzen, da das Bezugsobjekt gleichzeitig (teilweise) der Orientierungsrahmen des Verweisobjekts ist. Gestaltprinzipien schließen die Möglichkeit aus, daß das Umgekehrte ebenfalls gilt, nämlich daß das Verweisobjekt zur gleichen Zeit (teilweise) der Hintergrundrahmen des Bezugsobjekts ist.

Die wesentliche Schlußfolgerung dieses Abschnitts ist die, daß der Gebrauch der, vertikalen' Ausdrücke ,über' und ,unter' sich deutlich von dem unterscheidet, was wir hinsichtlich der beiden ,horizontalen' 
Dimensionen feststellen konnten. Die deiktische Verwendung von ,über" und ,unter" stellte sich als unabhängig von der Position des Betrachters/Sprechers bezüglich der betrachteten Szene heraus; worauf es ankommt, ist allein die wahrgenommene Vertikalität. Durch das Prinzip der kanonischen Orientierung ist der intrinsische Gebrauch von ,über ${ }^{\text {t }}$ und ,unter ${ }^{\text {w }}$ weitestgehend eingeschränkt. Wenn der Hintergrund, an den der Betrachter sich orientiert, für Verweisobjekt und Bezugsobjekt identisch ist oder in einer engen Beziehung steht, was den normalen Fall darstellt, dann ist intrinsische von deiktischer Verwendung nicht unterscheidbar. Intrinsischer Gebrauch von ,über" und ,unter' ist nur möglich, wenn es zu gleicher Zeit zwei verschiedene perzeptuelle Orientierungsrahmen gibt. Aber auch in diestir Fällen müssen Einschränkungen gemacht werden. Prinzipien der FigurGrund-Organisation folgend ist es beispielsweise auch dann nicht möglich, die Bedingungen der Umkehrbarkeit und Transitivität zu verletzen.

\section{Epilog}

Eine deiktische oder intrinsische Perspektive einzunehmen, sind grundsätzlich verschiedene Wege, um räumliche Verhältnisse in einer sprachlichen Äußerung adäquat auszudrücken. Eine wichtige Ursache für die Schwierigkeiten, denen wir uns beim Gebrauch dimensionaler Begriffe gegenübersehen, stellt zweifellos die Ko-Existenz deiktischer und intrinsischer Systeme räumlicher Referenz dar, Damit kommen wir zu der Frage der funktionalen Bedeutung dieser Duplizität (falls es eine solche Bedeutung überhaupt gibt) und zu der Frage, wie Sprachbenutzer mögliche Konfusionen zwischen beiden Systemen vermeiden können.

Empirische Hinweise darauf, welche Unterschiede im Gebrauch beider Systeme bestehen, gibt es nur sehr wenige. Die ausführlichste Untersuchung wurde von Ehrich (1985) durchgeführt. Sie bat Versuchspersonen, räumliche Anordnungen von Puppenmöbeln in eiñemi dafür konstruierten kleinen Raum zu beschreiben. Sie ordnete die einzelnen Möbelstücke so an, daß eine deiktische oder eine intrinsische Perspektive $\mathrm{zu}$ dem jeweils entgegengesetzten Gebrauch räumlicher Ausdrücke führte. Der wichtigste Befund war, $\mathrm{da} B$ das deiktische System bei weitem bevorzugt wurde. Ehrich argumentiert, da $B$ das deiktische System einfacher ist, wenn die Aufgabe darin besteht, eine komplexe räumliche Anordnung zu beschreiben. Eine intrinsische Beschreibung ist nur dann möglich, wenn alle Bezugsobjekte so wie Stühle und Schränke eine intrinsische Persyektive haben. Wenn das wie etwa bei Tischen und Lampenschirmen nicht der Fall ist, wird es notwendig, zwischen der Verwendung des deiktischen und des intrinsi- 
schen Systems abzuwechseln. Unter diesen Umstinden ist eine aus. schließliche Verwendung des deiktischen Systems einheitlicher. I ar. überhinaus solite nicht vergessen werden, dath dis intrinsische System nicht dafür geeignet ist, Schlubfolgerungen zu zichen, dic atu' Bezithungen der Umkehrbarkeit oder Transitivität batsieren. Lis wäre daher schwierig für einen Hörer, auf Grund einer intrinsischen Beschreibung eine komplexe räumliche Anordnung to rekonstruieren. Heim Bkschreiben komplexer räumlicher Anordnungen dürfte eine deiktische Strategie damit unter kommunikativen Gesichtsptunkten vortcilhah sein.

Diese Vorteile bei der Benutzung des deiktischen Systems sind leicht einzusehen. Man mag sich dann jedoch fragen, ob das intrinsi. sche System in anderen Situationen ähnliche Vorteile bietet. Wunderlich (1981) hat festgestell, daß3 Sprecher die intrinsische Perspektive hevos. zugen, wenn sie vor die Aufgabe gestellt werden, die Beriehung zwi. schen nur zwei sich nicht bewegenden Objekten zu besthreiben, wotki eines der Objekte eine eindeutige Vorderseite hatte, etwit cin (jebiade. Man könnte auch dann eine Bevorzugung der intrinsischen l'erspeklive erwarten, wenn der Sprecher weiB, dalB der Hörer Probleme dathei haben wird, die Perspektive des Sprechers hinsichtlich der a beschreibenden Szene zu rekonstruieren. Es ist unwahrocheinlich, dat in der Evolution die Entwicklung der Kommunikation über das Telefion antizipiert wurde, aber Unsicherheit hinsich tlich der Perspektive des Sprechers kann auch in anderen Situationen a lufkommen; ein evolutionarer Vorteil eines akustischen Kommunikationssystems wie der getprokhenen Sprache besteht genau darin, dab es benutat werden kann. wenn die Kommunikationspartner sich im Dunkeln befinden oder sich aus anderen Gründen nicht sehen können. Und das sind genaur die Situattivnen, in denen Unsicherheit hinsichtich der Perspektive des Splethers entstehen kann.

Allerdings sollten funktionale Vitklärungen für den Giehratteh des deiktischen gegenüber dem intrinsischen Systems mit Vorsicht hetriuthtet werden. Wir konnten feststellen, dati sich Personen stark darin unterscheiden, ob sie eine deiktische oder intrinsische Perspektive be* vorzugen. Die Ursachen für diese Prälerenzen sind noch nicht gettairt, aber es spricht einiges dafür, dalb es eine Beziehung zur Hündigketi gibt - (latente) Linkshänder benutzen die intrinsische Perspektive etwas stärker als Rechtshänder (vg!. Levelt, 1982).

Die Wahl eines intrinsischen oder deiktischen Weges zur Hestitreibung räumlicher Relationen hängt auf jeden Fall von mehreren bak to. ren $a b$, und der Hörer wird auf der Grundlage des nichtspratithlithen Kontexts oft nicht in der Lage sein vorherzusagen. von welcher f'stspektive der Sprecher ausgeht. Helfen Sprecher ihren Hörern herauszllfinden, welches System sie benutzen? Von Zeit zu Zeit markiert tin 


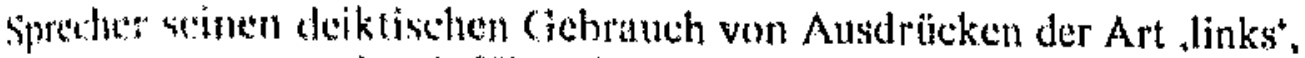
sechts", vor etc. durch Hinzulügungen wie "auk meiner Sicht " oder "wena man es von hier sieht". Aher das ist eher die Ausnathme als die Regel. In vielen Sprachen gibl es linguistische Mittel, um ąusschlie:Blich dic Verwendung des intrinsisehen Systems zu signalisieren. Ehrich (1985) hat festgestelit, dab,, u ihrer Linken' sich nicht neutral verhält hezuglich der firage, ob ein Sprecher eine intrinsische oder deiktische Perspektive cinnimmt, wahrend ,links von ihr ${ }^{2}$ in dieser Beziehung neutral ist, nu ihrer linkent kann nur intrinsiseh verwendet werden. Wunterlich (1981), Hill (1982) und Levelt (1982) diskutieren andere Beispicic in verschiedenen Sprachen. Obwohl Sprachen Mittel bureststellen. um die cigene perspektive anzuzeigen, werden diese Mittel kaum adtatguat genutzt. Offensichtlich vertrauen Sprachbenutzer in hohem Mabe der Intelligenz. und Kooperationsbereitschaft ihrer Kommunikationspartner.

\section{Lituratut}

(Ifork, If If (107) Space, time, semanticy, and the thild. In T. U. Moore (Ed,)

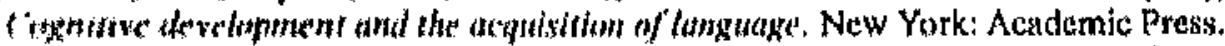

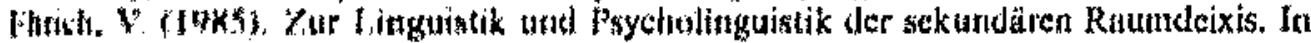

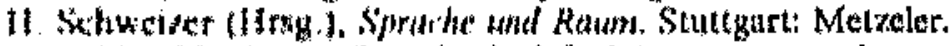

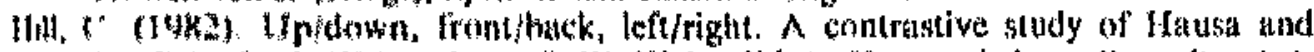

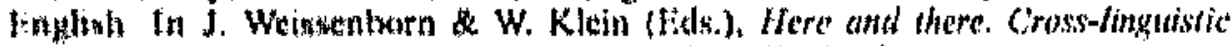

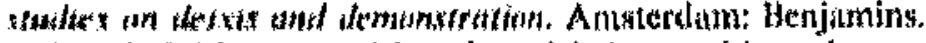

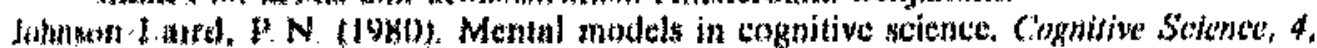
$11 \cdots 115$

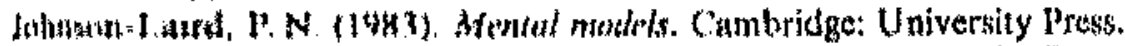

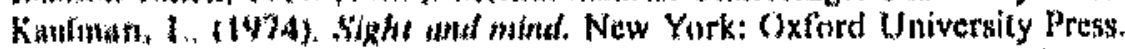

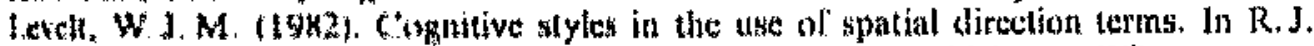

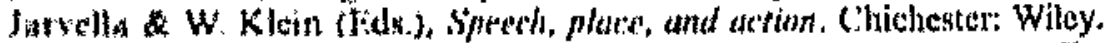

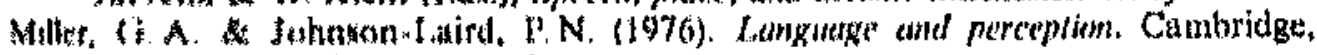

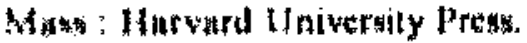

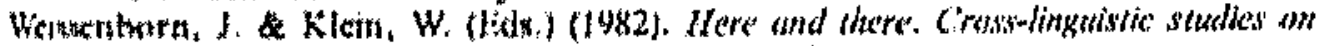

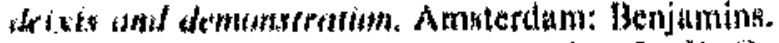

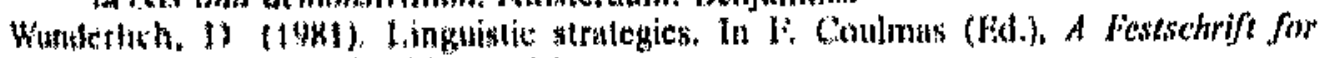

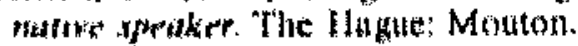

